\title{
Évolution sur 30 ans des pratiques en fin de vie en réanimation néonatale en France vue par un palliatologue
}

\section{Thirty years of evolution of neonatal reanimation end of life care in France: a} palliatologist's point of view

Raphaël Alluin $^{\mathrm{a} *, 1}$, Rachel Vieux $^{\mathrm{b}}$, Prisca Martini ${ }^{\mathrm{c}}$

\author{
${ }^{\text {a }}$ Service de soins palliatifs, hôpital de Hayange, CHR de Metz-Thionville, 1-3 rue du Friscaty, 57100 \\ Thionville, France \\ ${ }^{\mathrm{b}} \mathrm{CHU}$ de Besançon, 25000 Besançon, France \\ ${ }^{\mathrm{c}}$ Service de néonatologie, hôpital de Hayange, CHR de Metz-Thionville, 1-3 rue du Friscaty, 57100 \\ Thionville, France
}

\footnotetext{
*Auteur correspondant ; e-mail : ralluin@gmail.com

${ }^{1}$ Photo
}

Titre court :

Évolution sur 30 ans des pratiques en fin de vie en néonatologie en France 


\section{Introduction}

Cet article présente l'évolution de la prise en charge de la fin de vie en néonatologie en France depuis les années 1980 jusqu'à nos jours. Il retrace les différents courants de pensée et de prise en charge qui se sont succédés avec les années, éclairés par les publications scientifiques et les avis éthiques rendus par le Comité consultatif national d'éthique pour les sciences de la vie et de la santé (CCNE) au cours du temps. Il s'ouvre sur des perspectives de recherches intéressantes mais difficiles sur nos pratiques et la prise en charge de la fin de vie en néonatalogie.

\section{Prise en charge de la fin de vie en néonatalogie en France depuis les années 1980 : revue de la littérature et discussion}

La réanimation néonatale s'est grandement modernisée depuis les années 1980. Cette évolution, qui se poursuit depuis, a permis de diminuer de façon importante la morbimortalité des nouveau-nés prématurés en repoussant les limites de la médecine [1]. Elle a généré dans son sillage un ensemble de situations complexes et questionnantes. Les acteurs de la médecine néonatale ont ainsi été confrontés à de nouvelles limites : ils pouvaient sauver certaines vies mais ne pouvaient garantir à tous une vie sans séquelles, quelle qu'en soit la gravité [2]. La médecine devenait alors génératrice de handicap. Cela était particulièrement vrai pour les enfants nés dans ce que l'on appelle communément la zone grise, actuellement comprise en France entre 24 semaines d'aménorrhée (SA) et 25 SA plus 6 jours. Si ces bornes de la zone grise peuvent varier dans le temps d'un centre à un autre et particulièrement d'un pays à un autre [3], au-delà de la borne supérieure, invariablement les décisions sont moins sujettes à débat, puisque cette dernière tient toujours compte d'un pronostic considéré comme nettement meilleur. Toutefois il est vrai que même pour des enfants au-delà de ce terme, des dilemmes éthiques peuvent persister du fait de la survenue de complications au décours de la réanimation qui peuvent alors remettre en cause le pronostic initial et la pertinence de la prise en charge. De même, le problème des enfants atteints de pathologies malformatives congénitales pour lesquelles la grossesse a été menée à un terme avancé se pose, la grande variabilité de ces dernières situations amenant à des évaluations individualisées.

La pratique de «l'arrêt de vie » a alors vu le jour dans certaines équipes [4]. Elle faisait suite à une réflexion pluridisciplinaire de l'équipe soignante qui aboutissait à estimer que les traitements étaient devenus disproportionnés compte tenu de la lourdeur de ceux en place ou à entreprendre, faisant basculer la prise en charge dans l'obstination déraisonnable. Cette disproportion pouvait faire suite à l'établissement d'un diagnostic et donc d'un pronostic à l'issu d'un bilan complet, ou faire suite à une complication sévère au décours de la réanimation. C'est souvent la projection d'une qualité de vie future a priori très mauvaise qui faisait basculer, dans l'esprit des soignants, la prise en charge dans l'obstination déraisonnable. Ces pratiques d'arrêt de vie ont été analysées en profondeur par différents chercheurs notamment en sociologie. Leurs travaux publiés sous forme d'ouvrage ou d'articles 
retracent le processus décisionnel dans ce qu'ils appellent « les contextes de choix dramatiques », c'est-à-dire les situations où le pronostic vital de l'enfant est engagé [5-7]. L' « arrêt de vie » consistait alors à l'utilisation de médications visant à interrompre volontairement et activement la vie chez un sujet ne dépendant pas (ou plus) de traitements de support vital [8]. A contrario de l'emploi fréquent de ce terme « arrêt de vie », le mot euthanasie n'apparait pas ou peu dans la littérature scientifique puisque dans ce cadre précis il n'est pas possible au patient nouveau-né de faire une telle demande. Toutefois certains considèrent que par extension l'avis des parents équivaut à celui de l'enfant et parlent alors d'euthanasie à la demande des parents comme le Pr Verhagen dans le protocole Groningen [9].

Bien que cette pratique soit l'aveu d'un échec de la réanimation pour les équipes, et de fait une faiblesse dans la toute-puissance médicale, elle était aussi le symbole de leur désir de maitrise, en gardant la main jusqu'au bout, par la provocation du décès selon le timing choisi par l'équipe. Il semblait impossible pour les équipes de laisser faire la vie et d'accepter une évolution incertaine, de se contenter d'accompagner l'enfant dans son évolution naturelle comme l'aurait proposé la médecine palliative. Il n'existait que deux résultats pour ces équipes, un enfant vivant en bonne santé ou du moins avec des séquelles acceptables pour l'équipe soignante et les parents ou alors un enfant mort durant la prise en charge. Une troisième voie médiane ne semblait pas possible.

L'arrêt de vie trouvait sa justification dans les principes de base de l'éthique principiste, notamment la bienfaisance de la réanimation dite d'attente qui permettait de voir venir afin d'évaluer le potentiel du nouveau-né en dehors de l'urgence de la naissance, et souvent plusieurs semaines après. La non-malfaisance justifiait également cette option de la pratique de l'arrêt de vie, en ne laissant pas perdurer une situation jugée inacceptable [10]. Ces justifications ne permettent cependant pas de gommer tout questionnement, voire toute ambivalence. Un problème qui se pose ainsi par cette approche est l'absence de limite claire entre la réanimation justifiable et justifiée et l'obstination déraisonnable. Quelle est alors la limite entre les séquelles acceptables et celles qui sont inacceptables ? En effet, le pronostic des enfants prématurés dépend non seulement de facteurs bien connus dont le terme de la grossesse, le sexe, la corticothérapie anténatale, le lieu de naissance [11] ; mais ce pronostic évolue également rapidement d'un jour à l'autre, rendant toute projection de vie future hasardeuse pour les plus fragiles d'entre eux. La subjectivité des équipes semblent donc avoir une place importante dans ces situations.

Cette pratique d' " arrêt de vie » est cependant restée d'usage jusqu'au début des années 2000, et la notion « d'exception d'euthanasie » évoquée par le CCNE dans son avis $n^{\circ} 63$, a conduit ainsi certaines équipes à justifier les arrêts de vie comme autant de situations exceptionnelles [12]. Ces décisions apparaissaient, au terme d'une discussion pluri- ou interdisciplinaire, comme la moins mauvaise solution pour respecter l'enfant pour lequel les soignants n'avaient plus l'espoir d'un 
développement sans trop de séquelles. Toutefois, une des critiques faite à cette pratique est le caractère majoritairement subjectif des arguments utilisés, particulièrement sur la notion de handicap qu'aucune recommandation ne définit, tout comme le seuil de handicap lourd reste abscond [3]. Ces enfants ne vivaient que grâce à la réanimation dont ils avaient bénéficié mais c'est elle aussi qui avait fait d'eux des enfants handicapés. Il apparait donc ici que les réanimateurs en repoussant les limites de la médecine et en sauvant des vies à l'échelle d'une population créaient autant de situations singulières inextricables et sources de souffrance.

En outre, ce qui complexifie davantage ces questions épineuses est l'absence de connaissances médicales des parents ne leur permettant de projeter le futur de leur enfant qu'à partir des informations délivrées par les soignants relative à leur vision de la qualité de vie future de cet enfant [13]. Par ailleurs, ces informations sont basées sur des données issues de populations d'études. Or les probabilités à l'échelle d'une population sont généralisables à l'échelle populationnelle mais ne permettent pas de prédiction précise à l'échelle singulière. Cela bien sûr ne fait qu'augmenter l'incertitude.

Dans son avis $n^{\circ} 65$, le CCNE a remis en cause le concept de réanimation d'attente qualifié « d'acharnement thérapeutique initial », et mis l'accent sur la nécessité de prévenir la survenue des situations de détresse vitale à la naissance, en critiquant également la quête de la performance en néonatalogie [14]. Pour le CCNE en effet, cette recherche constante de l'amélioration des techniques pour réanimer des enfants de poids et d'âge gestationnel toujours plus faibles serait pourvoyeuse de situations dramatique sur le plan humain, et dans ces situations, il a qualifié l'arrêt de vie de «grave transgression ». Dans un des nombreux articles tirés de l'étude Épipage il était constaté que près de $50 \%$ des morts d'enfants prématurés faisaient suite à une procédure de limitation ou d'arrêt de traitement [15]. Cela met donc en lumière le taux de mortalité important après la réanimation d'attente mais l'étude ne donnait pas de détails sur les critères d'évaluation amenant à ces décisions de limitation et d'arrêt des traitements de réanimation.

Le CCNE a également ouvert le débat au niveau sociétal par rapport aux considérations des professionnels quant à la qualité de vie dite médiocre pour ces enfants lourdement handicapés en rappelant que la société n'a pas su réaliser l'investissement nécessaire sur le plan humain et financier pour l'intégration de ces enfants atteints de handicap(s). Cela a par la suite été repris par d'autres auteurs $[3,16,17]$. Il est à noter également l'absence d'étude de qualité de vie pour ces enfants et leurs familles.

Cette réorientation du CCNE entre l'ouverture à « l'exception d'euthanasie » (dans son avis $n^{\circ} 63$ ) et une recherche d'une réanimation plus raisonnée en amont (dans son avis $n^{\circ} 65$ ) avec un rappel de la qualité et de la valeur de la vie des enfants handicapés faisait suite à la parution des résultats de la première étude Épipage [14]. Cette étude a mesuré de façon objective, par un suivi 
longitudinal, la morbimortalité des enfants nés prématurés, mettant en évidence une nette amélioration de celle-ci, tous termes de grossesse confondus, sur les dernières décennies du fait des progrès médicaux réalisés. Elle a aussi montré que ces enfants avaient moins de séquelles et un besoin moins important de suivi médical que communément admis. Cela laissait entrevoir une qualité de vie potentiellement meilleure que celle projetée pour eux par les professionnels de santé ainsi qu'un poids familial moins lourd pour ces enfants. Or si la qualité de vie de ces enfants s'améliore (nous le supposons au vu des résultats d'Épipage) la légitimité du recours à des pratiques transgressives diminue d'autant pour ces enfants handicapés.

Dans ces décisions, les parents, atteints par la prématurité de leur enfant se rangeaient en grand nombre à l'avis des professionnels. Or, au cours des entretiens d'information des parents il semble que deux profils de néonatalogistes existent : ceux qui informent de façon claire et loyale les parents et les laissent choisir et assumer la décision, et ceux qui les orientent fortement en leur proposant une alternative comme meilleure que la/les autre(s) [18]. Cette dernière attitude tendrait à diminuer le sentiment de culpabilité des parents, notamment en cas de complication(s) survenant pour leur enfant. Pour autant, quelle que soit l'attitude médicale, il est parfois impossible aux parents de poser un choix dans une telle situation nouvelle et sidérante [19]. En ce qui concerne la décision, celleci reste médicale d'après de la loi, mais dans la pratique les médecins recherchent l'adhésion des parents pour des choix aussi cruciaux que la décision d'arrêter ou pas une réanimation ou de permettre ou non la survie d'un enfant handicapé. Bien que l'intérêt premier soit celui de l'enfant certains médecins considèrent que ce sont les parents qui porteront le poids de cet enfant toute leur vie et de fait, que la décision leur revient surtout dans les situations tangentes.

Au cours du temps, la pratique de l'arrêt de vie est officiellement devenue minoritaire, voire inexistante. De manière concomitante, la loi du 4 mars 2002 relative aux droits des malades et à la qualité du système de soins et celle de 2005 relative aux droits des malades et à la fin de vie ont été votées, et leurs décrets d'application publiés. Ces deux lois offrent la possibilité de mettre en place un projet de soins palliatifs dans la période péri- et néonatale. Elles constituent ainsi une alternative légale et éthique à ces « arrêts de vie » grâce à l'obligation d'éviter toute obstination déraisonnable par la possibilité de limitation ou d'arrêt des thérapeutiques. La limitation est la possibilité de ne pas faire plus, ou de ne pas réutiliser des thérapeutiques qui ont déjà été utilisées mais que l'amélioration de l'état clinique du patient a permis d'arrêter. L'arrêt des thérapeutiques est la décision à un moment précis d'arrêter tous les traitements non symptomatiques, notamment les traitements de support vital, en allant jusqu'à l'extubation si nécessaire. L'objectif de l'arrêt des thérapeutiques est de mettre fin à une réanimation ou un traitement de support vital en cours, car ils ont été jugés disproportionnés ou futiles dans la situation. Cet arrêt des traitements de support vital peut permettre le décès du patient du fait de sa maladie. A noter qu'il existe tout de même des cas de patients survivant aux arrêts de traitements de support vital. La médecine palliative a ouvert également à la possibilité d'administrer 
des thérapeutiques sédatives ou analgésiques dans l'intention de soulager le malade aux posologies requises par l'état du patient, et ce quelles qu'en soient les conséquences en terme vital, ces thérapeutiques pouvant à certaines doses abréger la vie du patient. Le principe du double effet consiste à administrer un traitement pour soulager le patient d'un symptôme même si cela a des conséquences néfastes pour lui, dès lors que l'intention unique du prescripteur est de soulager la souffrance du patient. Il apparait donc avec cette nouvelle façon de faire que la temporalité de la fin de vie est très différente. Ici le décès n'est plus provoqué à un moment précis mais permis, celui-ci survenant alors à un moment non calculé. Un temps d'agonie survient donc et la médecine palliative prend ici sa place pour transformer ce temps de mort en temps de vie en réalisant un accompagnement de l'enfant et de sa famille. En outre, ces enfants dont la vie est menacée (risque important de décès mais aussi possibilité d'une survie) peuvent survivre si leur état clinique se stabilise et atteindre l'âge adulte, ce qui n'était pas possible avec les « arrêts de vie ». Cette pratique ouvre donc la voie à la survie d'enfants atteints de handicaps.

La littérature traduit clairement cette évolution des pratiques décrites ci-dessus, de l'arrêt de vie décidé par l'équipe soignante sans réelle implication des parents, «pour les préserver de toute culpabilité », vers la pratique des soins palliatifs en néonatalogie avec une intégration des parents dans les décisions de projet thérapeutique, projet de vie, concernant l'enfant [4, 10,20]. La mise en place des soins palliatifs n'a néanmoins pas résolu toutes les situations éthiquement questionnantes en néonatalogie [20, 21]. Ainsi certains auteurs défendent toujours la possibilité de réaliser des euthanasies dans des conditions très particulières et exceptionnelles, même si ces euthanasies n'en affichent pas clairement le nom. Elles sont alors nommées « accompagnement actif », « sédation terminale » ou encore « écourtement du temps palliatif » lorsque ce dernier est considéré trop long et générateur de souffrance [20]. Dans ces pratiques l'utilisation du double effet n'a plus pour intention première de soulager mais de ne pas permettre la survie, voire d'entraîner la mort.

Les articles les plus récents font état d'une disparité des pratiques en France dans le cadre des soins palliatifs. Mazille et al. ont noté dans leur unité, une pratique des limitation ou d'arrêt des thérapeutiques dans $50 \%$ des décès de la période néonatale [20]. Les auteurs ont décrit une augmentation des antalgiques ou des sédatifs chez $19 \%$ des enfants en cas de survenue de gasps ou d'agonie prolongée. Ils ont présenté une procédure collégiale transparente et retranscrite dans le dossier concernant les situations de limitation ou d'arrêt des thérapeutiques ou de naissance en soins palliatifs, avec une implication forte des parents qui sont présents auprès de leur enfant dans $75 \%$ des situations de fin de vie. En revanche, ils ont rapporté l'absence d'arrêt de nutrition et hydratation artificielles du fait de la souffrance et du questionnement que cela engendre pour les équipes, cette pratique étant décrite comme minoritaire en France et moralement discutable. La vision de l'enfant mourant de dénutrition de façon lente et progressive est en général insoutenable, posant la question du sens de ce temps de vie et entraînant une grande souffrance des soignants et des proches [18]. Ils 
considèrent souvent que cela revient à laisser l'enfant mourir de faim et non pas de dénutrition. Le cas se pose aussi des enfants ayant une succion spontanée permettant des apports caloriques certes minimes, mais suffisants pour mourir lentement au terme d'une cachectisation importante, ou pour survivre et grandir avec un handicap lourd bien qu'une décision de limitation ou d'arrêt des thérapeutiques ait été prise en période néonatale [21]. Dans ce cas, certains auteurs sont partisans de la réalisation d'une sédation terminale de façon systématique, concomitante de l'arrêt de nutrition et d'hydratation pour limiter les souffrances, notamment l'angoisse éventuellement générée par une sensation de faim $[22,23]$. Cette pratique se basait sur la législation, avec la loi Leonetti qui permettait une sédation pour souffrance réfractaire et rejoint la nouvelle loi Claeys-Leonetti qui permet la réalisation d'une sédation profonde et continue jusqu'au décès notamment en cas d'arrêt de traitement de support vital (dont la nutrition et l'hydratation) [24,25].

Le questionnement quant à l'objectif est cependant vif dans ces situations difficiles et ouvre au débat sur la légitimité de telles pratiques. L'objectif est-il en effet de soulager une souffrance avérée ou supposée, ou d'éviter que l'enfant puisse survivre à la décision de limitation ou d'arrêt des thérapeutiques [20] ? Une étude menée par le Centre d'éthique clinique de Cochin sur les pratiques d'arrêt de nutrition et d'hydratation a montré trois profils dans les accompagnements [26] : le premier était un respect strict de la vie du patient en laissant à la nature le choix de survie de l'enfant qu'on laissait téter. Le deuxième profil correspondait aux soignants aidant activement l'enfant à mourir et le troisième profil correspondaient aux soignants cherchant une voie médiane d'un accompagnement digne de la fin de vie du patient. Ce dernier profil de soignant semblait offrir des soins de confort adaptés à la situation du patient, ils accompagnaient le patient vers son décès sans hâter ou retarder la mort, comme le proposent les soins palliatifs.

La projection d'une qualité de vie médiocre pour l'enfant, les lourdes conséquences envisagées pour les parents et la fratrie et le coût sociétal non négligeable prévisible nourrissent le débat [27]. Face à ce questionnement certains auteurs sont partisans d'une mise en place précoce de limitation ou d'arrêt des thérapeutiques avant les troisième ou quatrième jours de vie. Après ces premiers jours en effet, beaucoup de nouveau-nés sont déjà autonomes en termes de ventilation spontanée et d'absence de traitement de support vital, et ce souvent quelle que soit la gravité de leur détresse neurologique. Cette période initiale est qualifiée par ces auteurs de fenêtre d'opportunité. Ce laps de temps court implique de prendre une décision grave et irréversible pour l'enfant dans un contexte de grande incertitude puisqu'à cette période de vie le pronostic n'est pas encore établi de façon fiable [21]. Le risque est donc grand de ne pas permettre la survie d'enfants pour lesquels le développement positif aurait surpris, afin d'éviter la survie d'enfants atteints de handicap(s) potentiellement grave(s). 
En opposition à ce phénomène on peut citer l'article de Koper et al. de 2015 dans lequel les auteurs ont montré un allongement du temps de vie des enfants en soins intensifs avant leur décès aux Pays-Bas depuis la légalisation de l'euthanasie [28]. Bien que ce phénomène ne soit pas expliqué par l'étude, les auteurs concluaient à une attitude plus interventionniste qui laisserait plus sa chance aux enfants. Cela pourrait être lié à la possibilité d'utiliser l'euthanasie pour mettre fin à la situation en cas d'évolution clinique insuffisante.

D’autres éléments de la réflexion éthique semblent spécifiques à la néonatologie. Tel est le cas de la réanimation dite d'attente qui ouvre la voie à l'idée de pouvoir ultérieurement réparer les erreurs de la réanimation, en défaisant ce qui n'a été possible que grâce à la technicité de la prise en charge initiale [21]. Cette réanimation d'attente débute en salle de naissance quand l'état clinique du nouveau-né requiert des gestes de réanimation et lorsque cela survient de façon imprévue, en l'absence de discussion anténatale et de pronostic clairement établi. Dans ces situations le néonatologiste se base sur des éléments pronostiques connus tels le sexe, l'âge gestationnel et le poids à la naissance pour décider, souvent seul, et en situation d'urgence, de réaliser ou non des gestes de réanimation. Le doute sur l'avenir oriente de façon majoritaire le néonatologiste à envisager une évolution favorable et de fait à débuter des gestes de réanimation. Cette approche a été décriée par certains qui considèrent qu'elle serait réalisée à outrance et génèrerait de nombreux cas d'enfants avec de lourdes séquelles amenant à discuter d'une limitation ou d'arrêt des thérapeutiques. Dans ces situations très précises, où la survie initiale de l'enfant n'a été possible que grâce à la mise en place de techniques de réanimation, une partie des néonatologistes français estime qu'il est légitime plus tard, une fois le pronostic mieux établi, de pratiquer des « arrêts de vie » pour défaire ce qui a été fait et réparer une erreur qui n'aurait pas dû être, le pronostic étant plus défavorable qu'initialement supposé. En 2014, G. Moriette expliquait cette approche de la réanimation d'attente, tout en soulignant qu'il existe alors des pratiques de fin de vie différentes pour les enfants vivant de façon naturelle et ceux vivant à la suite de traitements de réanimation et donc de façon artificielle [21]. Cette approche est cependant considérée par certains néonatologistes comme plus « humaine » conformément à l'avis nº 63 du CCNE [10], et comme une interprétation de la loi «parmi d'autres possibles ». Par la suite, si le CCNE dans son avis $\mathrm{n}^{\circ} 121$ de juillet 2013 considère que l'euthanasie ne doit en aucune mesure être considérée comme une alternative à des soins palliatifs, il reconnaît qu'un cadre légal rigide ne peut pas rendre compte de l'ensemble de la complexité des situations de la fin de vie [29]. Il n'élimine pas la possibilité de faire le « choix d'une mort anticipée » mais le décrit comme un dernier recours ne pouvant faire suite qu'à un parcours de prise en charge palliative de qualité. Aussi «transgressif » que soit ce choix il ne doit pas être fait dans la clandestinité. Dans cet avis, un chapitre particulier est dédié aux «nouveau-nés, atteints de lésions cérébrales sévères et irréversibles ». Il est proposé d'utiliser la procédure collégiale de limitation ou d'arrêt des thérapeutiques mais également «que la loi soit interprétée avec humanité afin que, grâce à la manière de mener la sédation, le temps de l'agonie ne se prolonge pas au-delà du 
raisonnable ». Le CCNE impute une partie de ces situations aux « équipes » qui n'ont pas su « s'abstenir » à « la naissance ou même en anténatal ». Dans cet avis il est également fait mention du besoin de réaliser des études fiables dans le champ de la fin de vie, concernant les « situations limites », pour clarifier le débat.

Les résultats de l'étude Épipage 2, menée en 2011, ont montré une amélioration significative de la morbimortalité depuis Épipage 1, du fait de l'amélioration des techniques de réanimation [31]. Il est intéressant de se poser la question de l'impact des pratiques « d'arrêt de vie » sur les taux de survie sans séquelles présentés dans cette étude. En effet une forme de «sélection » des nouveau-nés survivants pourrait certes augmenter la mortalité, mais également diminuer la morbidité des survivants. On note une survie quasi nulle (0,7\% des enfants) avant 24 SA. Cela est très différent des taux de survie enregistrés dans d'autres pays occidentaux, très variables d'un centre à l'autre [31, 32]. Cela dénote le consensus français d'absence de prise en charge réanimatoire de ces nouveau-nés les plus immatures.

En 2014, P. Martini a confronté les résultats objectifs d'Épipage 2 au sentiment subjectif des professionnels (étudiants et seniors) concernant la morbimortalité des prématurés. Cette étude (présentée aux RNGO de 2014 et au congrès de la Société française de pédiatrie en 2015) a montré la surestimation par l'ensemble des professionnels de la périnatalité, de la mortalité, des séquelles et de leur sévérité chez les survivants, tout particulièrement pour les enfants nés avant $28 \mathrm{SA}$. Ce résultat pourrait amener à pondérer la nécessité de recourir à des arrêts de vie pour « le bien de l'enfant » bien que le point de vue du médecin ne soit pas le seul facteur décisionnel. Par ailleurs, cette étude montrait que la grande majorité des professionnels de la périnatalité était très réticente à la réanimation d'enfants nés avant 24 SA. Il convient toutefois de garder à l'esprit que cette étude s'attache au point de vue général des professionnels, c'est-à-dire que les questions ne portaient pas sur des cas précis mais sur une réflexion pour une population de patients. Il est toujours délicat de transposer une réflexion faite pour une population à un individu donné dans la pratique courante.

De façon plus essentielle, ces pratiques interrogent sur le sujet du bien recherché, à savoir s'il s'agit de l'enfant, des parents, des soignants ou de la société. La société française reste jusqu'à présent assez timorée dans ce qu'elle développe comme moyens permettant une réelle intégration des enfants porteurs de handicap(s). Ces éléments posent la question du pourcentage et du type de séquelle(s) à partir desquels il serait légitime aux soignants, et par eux à la société, de déclarer que le mieux pour l'enfant serait d'être mort [33]. Car nous ne parlons pas ici d'enfants en état végétatif chronique, mais bien d'enfants pouvant développer pour la grande majorité une vie sociale, que l'on peut a priori considérer de qualité, si on leur en donne les moyens. 


\section{Conclusion}

Tout cela note bien que la néonatalogie est une spécialité difficile notamment par ces considérations éthiques et ses enjeux multiples en situation de grande incertitude pronostique. Elle est en évolution constante, aux pratiques diverses à travers les différents centres français et étrangers. Il persiste de nombreuses situations questionnantes, particulièrement face aux perspectives d'une vie dont on peut se poser la question de la « valeur » au vu de sa qualité. Le développement de la médecine palliative est grandissant mais sa pratique semble encore variée en fonction des interprétations de chacun des situations cliniques, des recommandations et du cadre légal. Comme tout médecin, les néonatologistes, tireraient un bénéfice d'une formation approfondie dans le champ de la médecine palliative et de la réflexion éthique, formation non dispensée à un niveau d'expertise au cours de la formation initiale. La complexité des situations concernées, leur singularité, l'importance des nuances pour les aborder nous conduit à justifier la réalisation d'un travail afin d'explorer et comprendre sur quels arguments sont prises les décisions de fin de vie en réanimation néonatale. Nous formulons l'hypothèse que ces situations, du fait de toutes les incertitudes qu'elles comportent et du défaut de formation dans ce domaine, seraient vécues avec difficulté. Cela ne faisant que s'aggraver avec l'angoisse de « vouloir bien faire » et la culpabilité que l'on peut éprouver dans une situation où l'on ne sait pas si ce que l'on fait est le mieux ou le pire, et où l'on tend le plus souvent à rechercher le moins mal. Dans ces situations où il n’y a, de toute façon pas de bonne solution, la difficulté pour les soignants est de trouver la moins mauvaise. La législation française interdisant toute forme d'euthanasie majore le silence de certains professionnels sur ce sujet.

\section{Remerciements}

Nous remercions les professeurs Aubry et Viallard ainsi que l'ensemble de l'équipe pédagogique et des étudiants de la promotion 2014-2015 du master 2 « Recherche clinique en médecine palliative : soins, éthique et maladies graves ».

\section{Financement}

Ce travail n'a reçu aucun support financier.

\section{Conflits d'intérêts}

\section{Aucun}

\section{Bibliographie}

[1] Healy P, Fallon A. Developments in neonatal care and nursing responses. Br J Nursing 2014;23(1):21-4.

[2] Manaouil C. Obstination déraisonnable et réanimation du nouveau-né. Med Droit 2012;2012(112):1-9. 
[3] Dehan M, Zupan-Simunek V, Vial M. Les enjeux éthiques de l'extrême prématurité. J Gynecol Obstet Biol Reprod 2004;33(1):94-8.

[4] Dehan M, Gold F, Grassin M, Janaud JC, Morisot C, Ropert JC, et al Dilemmes éthiques de la période périnatale : recommandations pour les décisions de fin de vie. Arch Pediatr 2001;8(4):407-19.

[5] Gisquet E. Vie et mort en réanimation néonatale. Les processus décisionnels en contexte de choix dramatiques. Paris : L’Harmattan. 2008. P. 284.

[6] Paillet A. Sauver la vie, donner la mort. Une sociologie de l'éthique en réanimation néonatale, Paris : La Dispute ; 2007. P. 286.

[7] Kentish-Barnes N. « Mourir à l'heure du médecin ». Décisions de fin de vie en réanimation. Rev Fr Sociol 2007;48(3):449-75. DOI : 10.3917/rfs.483.0449.

[8] Bétrémieux P, Gold F, Parat S, Caeymaex L, Danan C, de Dreuzy P et al. Palliative care in the neonatal period. Part one: general considerations. Arch Pediatr 2010;17:409-12.

[9] Verhagen E, Sauer P JJ. The Groningen Protocol - Euthanasia in severely ill newborns. N Engl J Med 2005;352:959-62. DOI: 10.1056/NEJMp058026.

[10] Dieh, R, Rayet I, Teyssier G. Décision de fin de vie dans un service de réanimation néonatale: pratiques et vécu des soignants. Ethique \& Santé 2007;4:213-7.

[11] Tyson J E, Parikh N A, Langer J, Green C, Higgins R D. Intensive care for extreme prematurity — Moving beyond gestational age. N Engl J Med 2008;358:1672-81. DOI: 10.1056/NEJMoa073059

[12] Anon. Avis sur Fin de vie, arrêt de vie, euthanasie. $N^{0} 63$ - 27 janvier 2000. Paris : Comité consultatif national d'éthique pour les sciences de la vie et de la santé ; 2000.

[13] Garel M, Séguret S, Kaminski M, Cuttini M. Problèmes éthiques posés par l'extrême prématurité : résultats d'une étude qualitative auprès des obstétriciens et des sages-femmes. Gynecol Obstet Fertil 2007;35:945-50.

[14] Anon. Réflexions éthiques autour de la réanimation néonatale. $N^{0} 65$ - 14 septembre 2000. Paris : Comité consultatif national d'éthique pour les sciences de la vie et de la santé ; 2000.

[15] Larroque B, Bréart G, Kaminski M, Dehan M, André M, Burguet A et al. Survival of very preterm infants: Epipage, a population based cohort study. Arch Dis Child Fetal Neonatal Ed. 2004;89(2):F139-44.

[16] Ropert JC. Les dilemmes éthiques des décisions de fin de vie en période périnatale. Arch Pediatr $2002 ; 9$ (Suppl1) :43-8. 
[17] Ropert JC. Les décisions de fin de vie en période périnatale : un débat professionnel, une question de société. Arch Pediatr. 2001;8:349-51.

[18] Payot A, Gendron S, Lefebvre F, Doucet H. Deciding to resuscitate extremely premature babies: How do parents and neonatologists engage in the decision? Soc Sci Med 2007;64:1487-500.

[19] Gisquet E, Friedberg E. End of life decisions: tragic choices in neo-neo-natalogy. Eur J Disabil Research 2011;5(1):26-36.

[20] Mazille N, Litzler-Renault S, Weider I, Donato L, Astruc D, Kuhn P. Soins palliatifs en néonatologie : analyse et évolution des pratiques sur 5 ans dans un centre de niveau 3. Arch Pediatr 2014;21(2):177-83.

[21] Moriette G. Limitations et arrêt de traitements chez le nouveau-né. Ethica Clinica 2014;6(74):6576.

[22] Béranger A, Boize P, Viallard ML. Étude pilote sur les pratiques de l'arrêt de la nutrition/hydratation artificielle en réanimation néonatale. Arch Pediatr 2014;21(2):170-6.

[23] Article R4127-37. Code de la santé publique

[24] Loi n 2005-370 du 22 avril 2005 relative aux droits des malades et à la fin de vie.

[25] Loi no 2016-87 du 2 février 2016 créant de nouveaux droits en faveur des malades et des personnes en fin de vie.

[26] Fournier V, Maglio M. L'arrêt d'alimentation et d'hydratation en contexte de fin de vie. Paris : Centre d'éthique clinique de Cochin; nd.

[27] Chan K, Ohlsson A, Synnes A, Lee DS, Chien LY, Lee SK et al. Survival, morbidity, and resource use of infants of 25 weeks' gestational age or less. Am J Obstet Gynecol. 2001;185(1):220-6.

[28] Koper JF, Bos AF, Janvier A, Verhagen AA. Dutch neonatologists have adopted a more interventionist approach to neonatal care. Acta Paediatr 2015;104(9):888-93. doi:

10.1111/apa.13050.

[29] Aubry R, Azoulay M, Beaufils F, Benmakhlouf A, Claeys A, Comte-Sponville A, et al. Avis n ${ }^{\circ}$ 121. Fin de vie, autonomie de la personne, volonté de mourir. Paris : Comité consultatif national d'éthique pour les sciences de la vie et de la santé ; 2013.

[30] Ancel PY, Goffinet F, EPIPAGE-2 Writing Group, Kuhn P, Langer B, Matis J et al. Survival and morbidity of preterm children born at 22 through 34 weeks' gestation in France in 2011: results of the EPIPAGE-2 cohort study. JAMA Pediatr 2015;169(3):230-8. 
[31] Rysavy MA, Li L, Bell EF, Das A, Hintz SR, Stoll BJ et al. Between-hospital variation in treatment and outcomes in extremely preterm infants. N Engl J Med. 2015; 372:1801-11.

[32] Serenius F, Sjörs G, Blennow M, Fellman V, Holmström G, Maršál K et al. EXPRESS study shows significant regional differences in $1 \square$ year outcome of extremely preterm infants in Sweden. Acta Paediatr 2013;103(1):27-37. DOI: 10.1111/apa.12421

[33] Chiswick M. Infants of borderline viability: Ethical and clinical considerations. Semin Fetal Neonat Med 2008;13:8-15. 